\title{
Characterisation and tissue distribution of the PISCF allatostatin receptor in the red flour beetle, Tribolium castaneum
}

\author{
Neil Audsley ${ }^{\mathrm{a}, *}$, Hans Peter Vandersmissen ${ }^{\mathrm{b}}$, Robert Weaver ${ }^{\mathrm{a}}$, Paulina Dani ${ }^{\mathrm{a}}$, June Matthews ${ }^{\mathrm{a}}$, \\ Rachel Down ${ }^{\mathrm{a}}$, Kristel Vuerinckx ${ }^{\mathrm{c}}$, Young-Joon Kim ${ }^{\mathrm{d}}$, Jozef Vanden Broeck ${ }^{\mathrm{b}}$ \\ ${ }^{a}$ The Food and Environment Research Agency, Sand Hutton, York YO41 1LZ, UK \\ ${ }^{\mathrm{b}}$ Molecular Developmental Physiology and Signal Transduction, KU Leuven, Naamsestraat 59, P.O. Box 02465, B-3000 Leuven, Belgium \\ ${ }^{\mathrm{C}}$ Insect Physiology and Molecular Ethology, KU Leuven, Naamsestraat 59, B-3000 Leuven, Belgium \\ ${ }^{\mathrm{d}}$ Gwangju Institute of Science and Technology, 261 Cheomdan-gwagiro, Buk-gu, Gwangju 500-712, Republic of Korea
}

\section{A R T I C L E I N F O}

\section{Article history:}

Received 29 March 2012

Received in revised form

28 September 2012

Accepted 29 September 2012

\section{Keywords:}

G protein-coupled receptor

Somatostatin

Neuropeptide

Juvenile hormone

\begin{abstract}
A B S T R A C T
The insect PISCF/allatostatins (ASTs) are pleiotropic peptides that are involved in the regulation of juvenile hormone biosynthesis, are myoinhibitory on the gut and the heart, and suppress feeding in various insects, but their roles in beetles are poorly understood. To provide further insight into the significance of PISCF/ASTs in beetles, the PISCF/AST receptor from Tribolium castaneum has been characterised and its tissue distribution determined. The biological activity of the T. castaneum PISCF/AST (Trica-AS) was also investigated.

The Trica-AS receptor shows high sequence homology to other insect PISCF/AST receptors, which are related to the mammalian somatostatin/opioid receptors, a family of $G$ protein-coupled receptors. The Trica-AS receptor was activated in a dose-dependent manner by both Trica-AS and T. castaneum allatostatin double C (Trica-ASTCC) as well as Manduca sexta-allatostatin (Manse-AS). Other allatoregulatory peptides (a FLG/AST, a MIP/AST and an allatotropin) and somatostatin ${ }^{14}$ were inactive on this receptor.

Receptor transcript levels in tissues, determined by qRT-PCR, were highest in the head and the gut, with variable amounts in the fat body and reproductive organs. There were measurable differences in receptor levels of the head, fat body and reproductive organs between males and females. There was also a widespread distribution of Trica-AS in various tissues of $T$. castaneum. The Trica-AS peptide precursor was most abundant in the head and there was a significant difference between levels in the heads and reproductive organs of males and females.

Whole mount immunocytochemistry localised Trica-AS in the median and lateral neurosecretory cells of the brain, in the corpus cardiacum and throughout the ventral nerve cord. The peptide was also present in midgut neurosecretory cells, but no immunostaining was detected in the reproductive organs or Malpighian tubules.

The widespread distribution of both Trica-AS and its receptor suggest this peptide may have multiple roles in beetles. However, Trica-AS had no effect on the spontaneous contractions of the gut or ovaries of T. castaneum but this peptide did stimulate the release of proteases from the anterior midgut of another beetle, Tenebrio molitor. The activation of the Trica-AS receptor by Trica-ASTCC implies a physiological role for this peptide in beetles, which remains to be identified.
\end{abstract}

Crown Copyright (c) 2012 Published by Elsevier Ltd. All rights reserved.

\section{Introduction}

Three neuropeptide families have been shown to have allatostatic activity in various insects, and are commonly known as the A, B and C-type allatostatins (ASTs), reviewed by Weaver and Audsley (2009). Recently, Coast and Schooley (2011) have recommended

\footnotetext{
* Corresponding author.

E-mail address: neil.audsley@fera.gsi.gov.uk (N. Audsley).
}

changes in nomenclature for these peptides, re-naming them as the FGLa/ASTs (A-type), the MIP/ASTs (B-type) and the PISCF/ASTs (Ctype). This nomenclature will be used throughout the manuscript.

The first insect PISCF/AST (Manse-AS) was identified from the tobacco hawkmoth, Manduca sexta through its inhibitory actions on juvenile hormone $(\mathrm{JH})$ synthesis by the corpora allata (Kramer et al., 1991). This 15 residue peptide is characterised by a blocked Nterminus (pGlu), a free C-terminus, and a disulphide bridge linking its two Cys residues at positions 7 and 14. Identical peptides have 
been characterised from other Lepidoptera, and orthologues of this peptide have also been characterised or deduced from genomic data in a variety of other insects, including the red flour beetle Tribolium castaneum (reviewed by Weaver and Audsley (2009); Veenstra (2009)). Unlike the FGLa/ASTs and MIP/ASTs, where multiple structurally related peptides are produced from their precursors, the PISCF/AST genes encode only a single peptide. Veenstra (2009) has reported a second gene in arthropods that encodes a PISCF/AST-related peptide, which was named allatostatin double C (ASTCC) and the genes encoding ASTCCs have also been identified in a variety of insects, including T. castaneum. However the physiological significance of the expressed ASTCC peptides remains to be determined.

The PISCF/ASTs inhibit JH biosynthesis in moths and mosquitoes, but do not appear to regulate $\mathrm{JH}$ in any other insects tested to date, including the fruit fly, Drosophila melanogaster (reviewed by Weaver and Audsley (2009)). These peptides have, however, been shown to have other effects, including the inhibition of spontaneous contractions of the foregut, the suppression of feeding activity in larval Lepidoptera (reviewed by Audsley and Weaver (2009)), and are myoinhibitory on the heart in D. melanogaster pupae (Price et al., 2002). In addition, when continually fed to aphids (Acyrthosiphon pisum and Myzus persicae) Manse-AS suppresses feeding resulting in mortality and reduced fecundity (Down et al., 2010; Matthews et al., 2010). The functions of PISCF/ ASTs in many insects, including $T$. castaneum, are poorly understood, but for some insects at least, it is clear that the primary role may not be the inhibition of JH biosynthesis (reviewed by Audsley and Weaver (2009), Weaver and Audsley (2009)).

Similarities between the invertebrate PISCF/AST and the vertebrate somatostatin precursors and receptors suggest these peptides have a common ancestor (Veenstra, 2009). Somatostatin is a tetradecapeptide that inhibits secretions (of neurotransmitters and hormones), cell proliferation and smooth muscle contractions, acts through a group of five G protein-coupled receptors (GPCRs) known as somatostatin receptors (SSTR) $1-5$, which are structurally related to the vertebrate galanin and opioid receptors. The SSTRs are expressed throughout the central nervous system and in peripheral tissues such as the pancreas, liver and gastrointestinal tract, reflecting the pleiotropic actions of somatostatin (reviewed by Moller et al. (2003)).

The GPCRs are transmembrane proteins that consist of an extracellular N-terminus, seven transmembrane $\alpha$ helices and an intracellular C-terminus. The binding of extracellular ligands to the receptor activates a guanine nucleotide binding protein (G-protein) initiating an intracellular second messenger cascade resulting in a cellular response, which ultimately regulates physiological and/or behavioural processes.

Receptors with structural homology to the SSTRs have been functionally characterised in insects, including $D$. melanogaster (Kreienkamp et al., 2002) and the mosquito, Aedes aegypti (Mayoral et al., 2010). These receptors are distributed in various tissues, particularly in the central nervous system (CNS), and are activated by PISCF/ASTs.

The aims of this study were to characterise the PISCF/AST receptor from $T$. castaneum and investigate its tissue expression and the biological activity of $T$. castaneum PISCF/ASTs (Trica-AS), to provide further insight into the role and significance of PISCF/ASTs in this insect.

\section{Materials and methods}

\subsection{Insects}

Tribolium castaneum larvae and adults were obtained from a colony maintained at the Food and Environment Research Agency,
Sand Hutton, UK. Adult beetles were sexed according to the morphology of the prothoracic legs; the presence of short bristles on the femur in males.

\subsection{Isolation of $m R N A$ and generation of $C D N A$}

Whole guts from last stadium $T$. castaneum larvae were dissected under $0.1 \mathrm{M}$ phosphate buffered saline (PBS; pH 7.2). Gut contents were removed, tissues were rinsed in fresh PBS, blotted to remove excess buffer, and immediately frozen in $1.5 \mathrm{ml}$ Eppendorf tubes on dry ice, then stored at $-70^{\circ} \mathrm{C}$ until required. Total RNA was extracted from $\mathrm{ca} .10 \mathrm{mg}$ gut tissues using a ZR Tissue and Insect RNA MicroPrep ${ }^{\mathrm{TM} K i t}$ (Zymo Research Corp., Irvine, CA, USA) according to manufacturer's protocols. A SMARTer ${ }^{\mathrm{TM}}$ RACE cDNA Amplification kit (Clontech, Mountain View, CA, USA) was then used to generate RACE ready cDNA.

Other tissues (fat body, reproductive organs) were also dissected and total RNA prepared as described above. Superscript III ${ }^{\circledR}$ reverse transcriptase (Invitrogen.com) was used to generate cDNA for real time (RT)-PCR, according to manufacturer's protocols.

\subsection{Genome screening and Primer design}

Hauser et al. (2008) identified the putative gene orthologue of the $D$. melanogaster PISCF/AST receptor in the T. castaneum genome (GLEAN_12842; accession number BK006110). The predicted T. castaneum PISCF/AST (Trica-AS) receptor gene, and the PISCF/AST receptor genes of D. melanogaster (DROSTAR 1, CG7285 and DROSTAR 2, CG13702; Kreienkamp et al., 2002) and Aedes aegypti (AeAS-CrA, AEL012356 and AeAS-CrB, AAEL012920; Mayoral et al., 2010) were manually aligned to reveal conserved and unique regions for primer design. Forward and reverse gene-specific (gsp) and nested PCR primers were designed using the Primer3 software package, version 0.4.0, hosted at the Whitehead Institute for Biomedical Research (http://frodo.wi.mit.edu/primer3/). Genespecific primers were selected to be $23-28$ nt in length, with 50-70\% GC content and a melting point greater than $65^{\circ} \mathrm{C}$. The PCR primers were custom synthesised by Eurofins MWG Operon (Edersberg, Germany). Primers were reconstituted in RNase/ DNase-free water, to a concentration of $100 \mathrm{pmol} / \mu \mathrm{l}$. The primers used to generate the full length Trica-AS receptor were:

\section{Forward primer CACCAGCCGTTGCACAATGAACGAAAGC} Reverse primer GTCTTGGACCACAACCGAACCTATCCAT

\subsection{Rapid amplification of $c D N A$ ends (RACE)}

The full length allatostatin receptor cDNA was produced using RACE ready CDNA and the SMARTer ${ }^{\mathrm{TM}}$ RACE cDNA amplification protocol with SMARTScribe ${ }^{\mathrm{TM}}$ Reverse Transcriptase, according to manufacturer's instructions (Clontech). A Biometra T3 thermocycler (Goettingen, Germany) was used for PCR reactions, using the following cycling conditions: $98^{\circ} \mathrm{C}$ for $60 \mathrm{~s}$, followed by 29 cycles of $98^{\circ} \mathrm{C}$ for $10 \mathrm{~s}, 68^{\circ} \mathrm{C}$ for $30 \mathrm{~s}$ and $72^{\circ} \mathrm{C}$ for $45 \mathrm{~s}$, then $72^{\circ} \mathrm{C}$ for $10 \mathrm{~min}$. The RACE products were separated using $1 \%$ agarose gels in TrisBorate EDTA buffer (Severn Biotech, UK) and visualised using Gel Red ${ }^{\mathrm{TM}}$ stain (Cambridge Bioscience, UK). Gel products were purified using the Wizard ${ }^{\circledR}$ SV Gel and PCR clean-up system (Promega) and sequenced using an Applied Biosystems 3730 DNA Sequencer (DNA Sequencing and Services, University of Dundee, UK). The PCR products were cloned, using the pcDNA ${ }^{\mathrm{TM}} 3.1$ Directional TOPO expression kit (Invitrogen), into the vector pcDNA ${ }^{\mathrm{TM}} 3.1 \mathrm{D} / \mathrm{V} 5-\mathrm{His}$ TOPO, which was then transformed into TOP10 E. coli. After culturing cells in LB medium containing $100 \mu \mathrm{g} / \mathrm{ml}$ ampicillin, 
plasmid DNA was purified using a QIAprep Spin Miniprep Kit (Qiagen, Crawley, UK). The orientation of the insert was verified by enzyme digestion (using BsrGI and Notl), and confirmation of full length Trica-AS Receptor by DNA sequencing. The cloned sequence was compared with human SSTRs and orthologues from $D$. melanogaster and $A$. aegypti using the alignment programme MEGA 5 (http://www.megasoftware.net/).

\subsection{Cell culture and receptor assays}

Human embryonic kidney cells (HEK293T) were cultured $\left(37^{\circ} \mathrm{C}\right.$, $5 \% \mathrm{CO}_{2}$ ) in monolayers in DMEM medium supplemented with $10 \%$ heat inactivated fetal bovine serum, $100 \mathrm{IU} / \mathrm{ml}$ penicillin and $100 \mu \mathrm{g} / \mathrm{ml}$ streptomycin (all: Sigma). Two receptor reporter assays were used (in different laboratories) to measure receptor activation by neuropeptides. Both these assays rely on receptor interaction with a promiscuous G-protein $\alpha$ subunit, either the murine $G \alpha 15$ or the human $\mathrm{G} \alpha 16$, both of which couple receptors to phospholipase C (Offermanns and Simon, 1995). This ultimately results in the release of $\mathrm{Ca}^{2+}$ from the endoplasmic reticulum, which can be measured as described below.

a. Fluo-4-AM assay. The pcDNA3.1D construct and a construct encoding the promiscuous G $\alpha 16$ were transfected into HEK cells using Fugene6 (Roche) according to the manufacturer's guidelines. Transfected cells were detached from the culture flask $48 \mathrm{~h}$ after transfection and transferred to 96 well plates at a confluence of approximately $70 \%$. After $24 \mathrm{~h}$, the cells were loaded with the $\mathrm{Ca}^{2+}$ reporter Fluo-4-AM (Molecular Probes) for $1 \mathrm{~h}$, after which the cells were washed with HBSS buffer supplemented with $5 \mathrm{mM} \mathrm{CaCl}_{2}$ and $10 \mathrm{mM}$ Hepes (Sigma). The cells were challenged with a dilution series of peptide ligand, dissolved in the same HBSS buffer. Excitation of the fluorophore was done at $488 \mathrm{~nm}$, fluorescence response was measured for $2 \mathrm{~min}$ at $525 \mathrm{~nm}$ using the FLEXStation (Molecular Devices). Data were analysed using Softmax Pro (Molecular Devices). As a negative control, mock-transfected cells were used.

b. Aequorin assay. The pcDNA3.1D construct and constructs encoding the promiscuous $\mathrm{G} \alpha 15$ and apoaequorin were transfected into HEK cells using Fugene6 (Roche) according to the manufacturer's guidelines. Transfected cells were detached from the culture flask $48 \mathrm{~h}$ after transfection, pelleted and resuspended, at a concentration of $5 \times 10^{6}$ cells $/ \mathrm{ml}$, in clear DMEM/F12 containing L-glutamine, 15 mM HEPES and 0.1\% BSA (BSA medium). Cells were loaded with $5 \mu \mathrm{M}$ coelenterazine by incubating for $4 \mathrm{~h}$ in the dark at room temperature whilst gently shaking. Cells were then diluted 10 -fold in BSA medium and incubated under the same conditions for a further $60 \mathrm{~min}$. Peptide solutions were diluted in BSA medium and $50 \mu \mathrm{l}$ aliquots dispensed into the wells of a white 96 multiwell plate. Light emission was recorded for $30 \mathrm{~s}$, after injection of $50 \mu \mathrm{l}$ of transfected cell suspension, using a Centro LB 960 microplate luminometer (Berthold Technologies, Bad-Wildbad, Germany). The cells were then lysed by injecting $0.3 \%$ Triton-X100 into each well, and light emission measured for a further $10 \mathrm{~s}$ to determine total cellular $\mathrm{Ca}^{2+}$ response. Mock transfected cells and BSA media was used as negative controls. The data files were exported into excel (Microsoft) for analysis.

\subsection{Real time $P C R$}

Primers for quantitative real time (qRT)-PCR were designed using Primer Express 3.0 (Applied Biosystems) for the Trica-AS receptor and peptide precursor genes. Using GeNorm
(Vandesompele et al., 2002), ribosomal protein (Rp)49 and RpS13 were selected as best reference genes out of five candidate reference genes, Rp49, RpS13, actin, ubiquitin and elongation factor $1 \alpha$. Primers were obtained from Sigma-Aldrich and their suitability for qRT-PCR was tested by means of melting curve and standard curve analyses, as well as gel electrophoresis of the PCR product. qRT-PCR was performed using Fast SYBR ${ }^{\circledR}$ Green Master Mix in a StepOne ${ }^{\mathrm{TM}}$ Plus System (both Applied Biosystems) following the manufacturer's instructions. The PCR protocol was: $95^{\circ} \mathrm{C}$ for $10 \mathrm{~min}, 40 \times$ (95 ${ }^{\circ} \mathrm{C}$ for $15 \mathrm{~s}, 60{ }^{\circ} \mathrm{C}$ for $1 \mathrm{~min}$ ). All samples were normalised against an external calibrator sample. Data were collected using StepOne ${ }^{\mathrm{TM}}$ Software v2.0 (Applied Biosystems) and plotted and analysed using Graphpad Prism5.

\section{Rp49 Fwd: TGGCAAACTCAAACGCAACT \\ Rp49 Rev: AGCGCCTACGAACCCTGTT \\ RpS13 Fwd: CCCCTTCACAAATAGGTGTCACA \\ RpS13 Rev: TTGCCTGTTACGAACCGAACTT \\ AS Rec Fwd: CAAACCGTCACCAACATGTACAT \\ AS Rec Rev: GGTATGCCGATGAGGAAGCA \\ AS Peptide Fwd: TTTATCTTCCTCATCGCCACTCT \\ AS Peptide Rev: TGAGACGCGTCCCCAAAG}

\subsection{Peptides}

The $T$. castaneum PISCF/AST (Trica-AS), deduced from the genome database (Weaver and Audsley, 2008), and the $M$. sexta PISCF/AST (Manse-AS) were synthesised by Cambridge Research Biochemicals, Billingham, UK. The T. castaneum allatostatin double C (Trica-ASTCC) and A. pisum myoinhibitory peptide (Acypi-MIP/ AST 8) were synthesised by Biomatik (Cambridge, Ontario, Canada). The FGLamide allatostatin from Periplaneta americana (Perpl-AST 6) was synthesised by the Advanced Biotechnology Centre, Imperial College, London, UK. Manduca sexta allatotropin (Manse-AT) and somatostatin-14 were purchased from Sigma, UK. The peptides were dissolved in water or $10 \%$ acetonitrile, and quantified using reversed phase-high performance liquid chromatography (RPHPLC). Sequences and alignments of peptides are shown in Table 1.

\subsection{Immunocytochemistry}

Tissues were carefully dissected from adult male and female T. castaneum in $0.1 \mathrm{M}$ PBS, and fixed overnight at $4{ }^{\circ} \mathrm{C}$ in $4 \%(\mathrm{w} / \mathrm{v})$ paraformaldehyde in $0.1 \mathrm{M}$ PBS ( $\mathrm{pH} 7.2$ ). Fixed tissues were washed 4 times in PBS for $15 \mathrm{~min}$, then incubated for 2 days at $4{ }^{\circ} \mathrm{C}$ with Manse-AS antisera (1:500 dilution) in PBS containing (v/v) $3 \%$ Triton X-100 (PBS-TX100) and 2\% goat serum (Sigma, UK). A control was performed by incubating fixed tissues in PBS-TX100 + goat serum without Manse-AS antisera. A further control was performed by pre-incubating (1:500 dilution in PBS-TX100 + goat serum) with

\section{Table 1}

Aligned amino acid sequences of peptides assayed on the Tribolium castaneum allatostatin receptor expressed in HEK cells. Tribolium castaneum PISCF-allatostatin (TricaAS) and allatostatin double C (Trica-ASTCC). Manduca sexta allatostatin (Manse-AS) and allatotropin (Manse-AT), Periplaneta americana allatostatin (Perpl AST 6), and Acyrthosiphon pisum myoinhibitory peptide (Acypi MIP 8). Amino acids identical to those of Trica-AS are highlighted in grey, $\mathrm{pE}=$ pyroglutamic acid, $\mathrm{a}=$ amide.

\begin{tabular}{ll}
\hline Peptide & Sequence \\
\hline Trica-AS & pESRYRQCYFNPISCF \\
Manse-AS & pEVRFRQCYFNPISCF \\
Trica-ASTCC & GHGSMSGQQKRVYWRCYFNAVTCF \\
Manse-AT & GFKNVEMMTARGFa \\
Perpl-AST 6 & ARPYSFGLa \\
Acypi MIP 8 & SWDNFQGSWa \\
Somatostatin-14 & AGCKNFFWKTSC \\
\hline
\end{tabular}


$100 \mu \mathrm{M}$ Trica-AS, prior to incubation with tissue. Tissues were washed $(4 \times 15 \mathrm{~min})$ in PBS-TX100 then incubated for a further 2 days with secondary antibody (Alexa Fluor 546 goat anti-rabbit $\operatorname{IgG}$ ) diluted 1:500 in PBS-TX100 + goat serum. After a further wash in PBS-TX100 $(4 \times 15 \mathrm{~min})$, tissues were mounted on microscope slides in Vectashield ${ }^{\circledR}$ mounting medium (Vector Laboratories, Peterborough, UK) for fluorescence microscopy, using a Leica DMLB microscope.

\subsection{Bioassays}

\section{a. Myoactivity}

Contractions of the gut and oviduct were measured using semiisolated preparations in a manner similar to those described by Matthews et al. (2008) for contractions of the lepidopteran foregut. Briefly, adult female beetles were pinned to a wax dish under a $100 \mu \mathrm{l}$ drop of beetle saline (mM: $\mathrm{NaCl}, 274 ; \mathrm{KCl}, 19 ; \mathrm{CaCl}_{2}, 9$; glucose, 5 ; Hepes, 5; pH 7.0). The elytra and wings were removed and the cuticle on the dorsal surface of the abdomen was opened and pinned back. The gut or ovaries were exposed by carefully removing any fat body. After rinsing with $2 \times 100 \mu$ of saline, the tissues were bathed in a fresh droplet of saline and contractions observed under a stereo microscope. Contractions (gut or oviduct) were counted over $2 \times 1 \mathrm{~min}$ (control) periods, prior to adding test solutions of Trica-AS, after which contractions were monitored for a further $2 \times 1 \mathrm{~min}$ periods.

\section{b. Protease assays}

The release of proteases from the anterior midgut of Tenebrio molitor larvae was determined by measuring total protease activity, using azocasein as a substrate.

Anterior midguts were removed, opened as flat sheets and incubated in $100 \mu \mathrm{l}$ beetle saline $+/-$ Trica-AS for $30 \mathrm{~min}$ at $30^{\circ} \mathrm{C}$. After gentle centrifugation, the supernatants were removed, and aliquots tested for protease activity, by incubating with $0.25 \%(\mathrm{w} / \mathrm{v})$ azocasein in $50 \mathrm{mM}$ phosphate buffer ( $\mathrm{pH}$ 5.7) containing $0.05 \%$ sodium dodecyl sulphate (total volume $40 \mu \mathrm{l}$ ) in Eppendorf tubes. The reaction was terminated by adding $30 \mu \mathrm{l} 10 \%(\mathrm{v} / \mathrm{v})$ trichloroacetic acid. After incubation on ice for $30 \mathrm{~min}$, tubes were centrifuged at $12,000 \times \mathrm{g}$ for $5 \mathrm{~min}$ at room temperature, and $60 \mu \mathrm{l}$ of the supernatant added to wells of a microtitre plate containing $40 \mu \mathrm{l}$ $1 \mathrm{M} \mathrm{NaOH}$. Plates were read at $405 \mathrm{~nm}$ on a Labsystems Multiskan MS plate reader (ThermoFisher Scientific, UK).

\section{Results}

\subsection{Identification of the Trica-AS receptor}

Using total RNA isolated from whole guts of T. castaneum larvae, and primers based on conserved regions of the D. melanogaster and A. aegypti PISCF/AST receptors, and on the coding sequences predicted from the T. castaneum genome, the cDNA, coding for the Trica-AS receptor was isolated and sequenced by reverse transcriptase PCR. The cDNA was amplified using forward and reverse primers directed at the $3^{\prime}$ and $5^{\prime}$ ends of the predicted coding sequence. An open reading frame of 1248 base pairs was detected, coding for a protein of 416 amino acids (see Supplementary Fig. 1). The deduced protein sequence including (predicted) seven transmembrane domains and a "DRY" motif, characteristic of rhodopsinlike GPCRs are shown in Fig. 1. The Trica-AS receptor (LOC659813) is located on chromosome 9 of the T. castaneum genome. The gnomon predicted genomic sequence (Loc 659813) shows two introns within the Trica-AS receptor gene, one between the TM6 and TM7 coding regions, and a second located close to the $3^{\prime}$-end, followed by a small coding region. However, results suggest that this second intron and small coding region are not part of the transcribed mRNA, and the $3^{\prime}$-end of the Trica-AS receptor mRNA terminates before this intron.

The sequence of the Trica-AS receptor is compared with the sequences of human SSTRs and orthologues from $D$. melanogaster and A. aegypti (Fig. 1), showing high overall amino acid similarity between these receptors, as indicated by the number of identical and conservatively substituted residues. The phylogenetic analysis between these receptors (Fig. 2) shows that the insect PISCF/AST receptors are closely related and cluster together, whereas the structurally related human SSTRs, form a separate cluster. Within the cluster of insect PISCF/AST receptors, the proteins from Diptera seem to be more related to each other than to the T. castaneum receptor, as would be expected.

\subsection{Activation of the Trica-AS receptor}

The Trica-AS receptor that was expressed in HEK cells transiently expressing the G $\alpha 16$ was activated in a dose-dependent manner by Trica-AS and Manse-AS (Fig. 3a). The $\mathrm{EC}_{50}$ value for Trica-AS was $12 \mathrm{nM}$, and for Manse-AS $20 \mathrm{nM}$. Mock-transfected HEK-293 cells did not respond to Trica-AS. Using cells that do not express $\mathrm{G} \alpha 16$, receptor activation could also be detected by $\mathrm{Ca}^{2+}$ measurement, indicating that the receptor naturally signals through $\mathrm{Ca}^{2+}$.

The Trica-AS receptor expressed in HEK cells transiently expressing $\mathrm{G} \alpha 15$ and aequorin was activated in a dose-dependent manner by Trica-AS and also by Trica-ASTCC (Fig. 3b), giving $\mathrm{EC}_{50}$ values of $25.0 \mathrm{nM}$ and $105.7 \mathrm{nM}$ respectively. In contrast, other allatoregulatory peptides (Perpl-AST 6, Acypi-MIP 8 and Manse-AT) and somatostatin ${ }^{14}$ had no effect, at doses up to $10 \mu \mathrm{M}$ (results not shown). Mock transfected cells did not respond to Trica-AS.

\subsection{Tissue localisation of the Trica-AS receptor}

The localisation and relative abundance of the PISCF/AST receptor transcript was determined by qRT-PCR. The highest transcript levels were in the head of both male and female beetles. Smaller amounts were present in the gut and testes, and variable levels were present in other tissues including the fat body and reproductive organs (Fig. 4). There was a significant difference between male and females in the levels of Trica-AS receptor in the fat body and the head ( $t$-test, $P<0.05$ ), but not in the gut. The relative amounts in reproductive organs were higher in testes compared with ovaries.

\subsection{Tissue localisation of Trica-AS}

\section{a. Quantitative RT-PCR}

Quantitative RT-PCR showed that the Trica-AS peptide precursor was present in greatest amounts in the head with lower transcript levels in the gut and fat body and testes (Fig. 5). Levels of Trica-AS transcript were significantly higher in the head of males, compared with females and in testes compared with ovaries ( $t$-test, $P<0.05$ ). Similar levels were present in the whole gut of both sexes.

\section{b. Whole mount immunocytochemistry}

Whole mounts of larval brains and retrocerebral complex treated with antisera raised against Manse-AS showed (weak) immunostaining in the median neurosecretory cells of the pars intercerebralis (Fig. 6a, arrow II), and in the lateral neurosecretory 


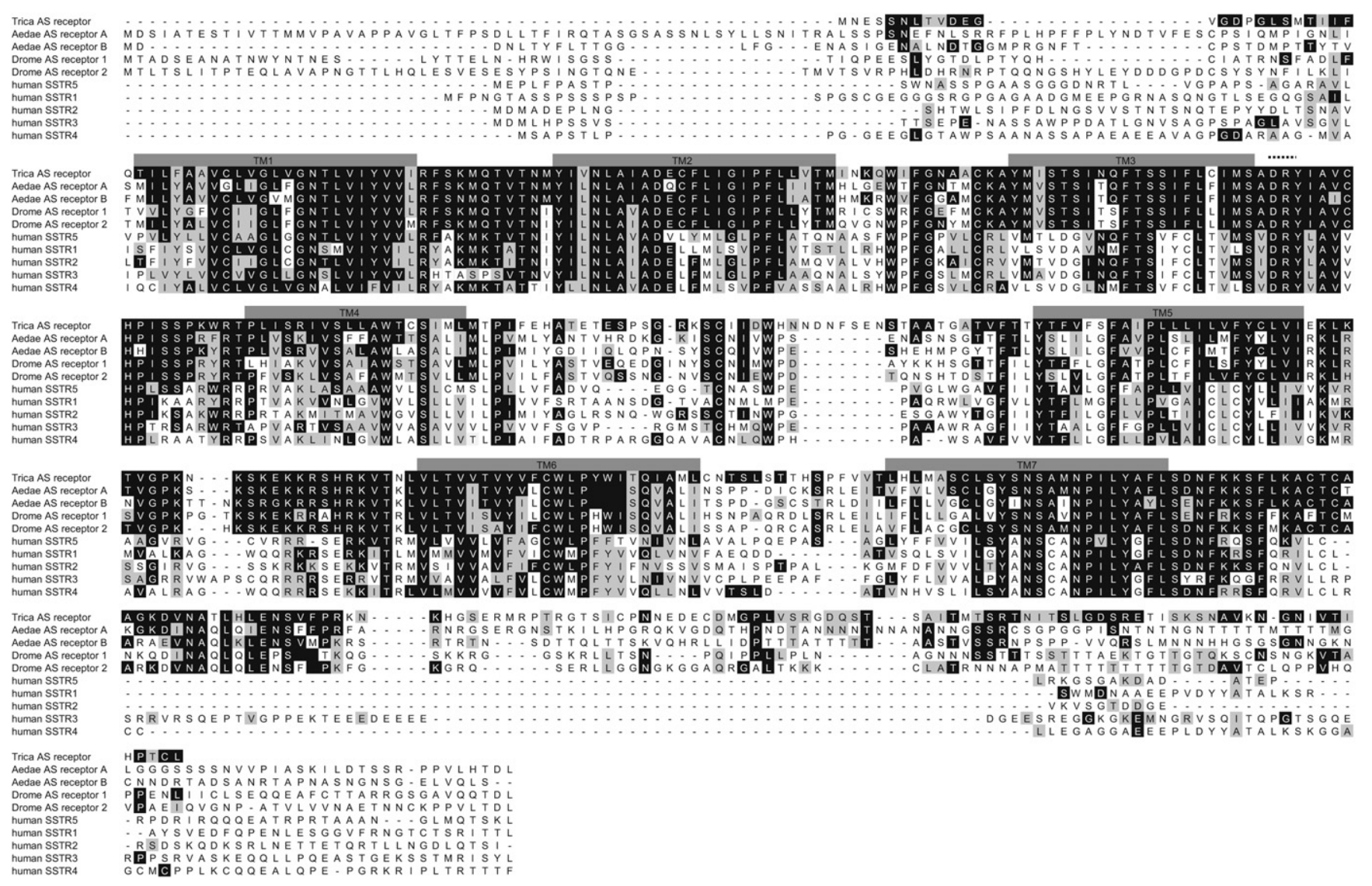

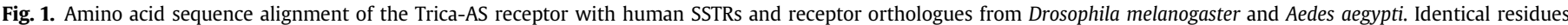

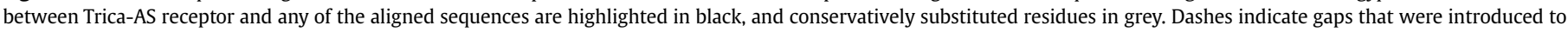

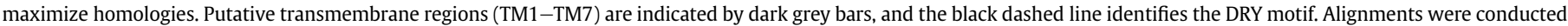
using the programme MEGA 5.

cells (arrow I) of the brain. Immunostaining was also present in the axons entering the corpus cardiacum (CC), giving rise to immunoreactive varicosities around the edge of this tissue, however, no immunostaining was apparent in the corpus allatum (CA; Fig. 6b).

Immunoreactivity to Manse-AS antisera was present in pairs of nerves in the ventral nerve cord, and in varicosities in all ventral ganglia (Fig. 6c, d). Two cells in the terminal abdominal ganglion also immunostained with Manse-AS antisera (Fig. 6e).

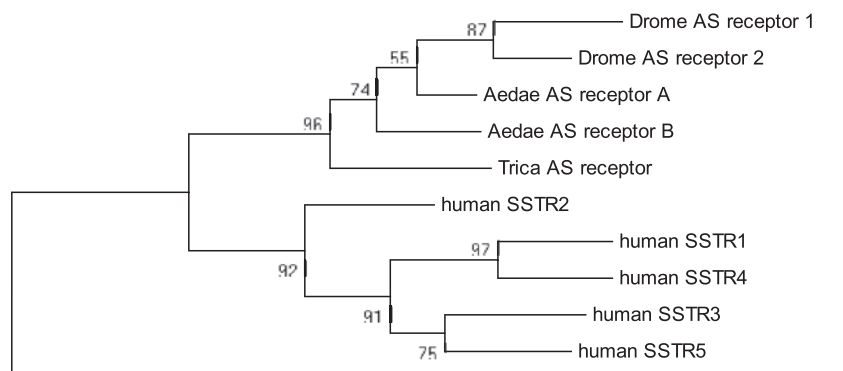

ก?

Fig. 2. The phylogenetic relationship between the characterised PISCF allatostatin receptor from Tribolium castaneum (Trica-AS receptor) orthologues of this receptor from Drosophila melanogaster; (Drome AS receptor 1; CG7285, and receptor 2; CG13702), Aedes aegypti (Aedae AS receptor A; AAEL012356, and receptor B; AAEL012920), human somatostatin receptors (SSTR1-5; NM 001049, NM 001050 NM 001051 NM 001052, NM 001053) and T. castaneum sex peptide receptor (EU106877). Phylogenetic analyses were conducted using the maximum-likelihood method with MEGA 5 software. Bootstrap values were calculated using 800 replicates.
The Trica-AS peptide was also localised in the anterior midgut neurosecretory cells (Fig. 7), but it was not detected in any other region of the gut, or nerves innervating various regions of the gut.

No immunostaining was detected in the reproductive organs, Malpighian tubules, in tissues incubated in the absence of primary antisera (results not shown), or in tissue pre-treated with primary antisera incubated with Trica-AS (Supplementary Fig. 2)

\subsection{Bioassays}

\section{a. Myoactivity}

The rectum appeared to be the only region of the gut to spontaneously contract, using semi-isolated preparations, and the rates of contraction were highly variable between individual insects. The mean control rate was $23.2 \pm 2.0$, ranging from 4 to 45 contractions/min $(n=30)$. Control rates were not significantly affected by the application of Trica-AS to gut tissues, even at high doses $\left(10^{-5} \mathrm{M}\right)$

Contractions of the oviduct were also variable, ranging from 2 to 24 contractions $/$ min $($ mean $=9.8 \pm 0.6, n=81$ ). At concentrations between $10^{-9}-10^{-6} \mathrm{M}$, Trica-AS had no effect on control rates of oviduct contractions.

\section{b. Protease secretion}

The release of proteases from the anterior midgut of T. molitor was stimulated by Trica-AS. The total protease activity (using azocasein as substrate), measured as absorbance at $405 \mathrm{~nm}$, increased 

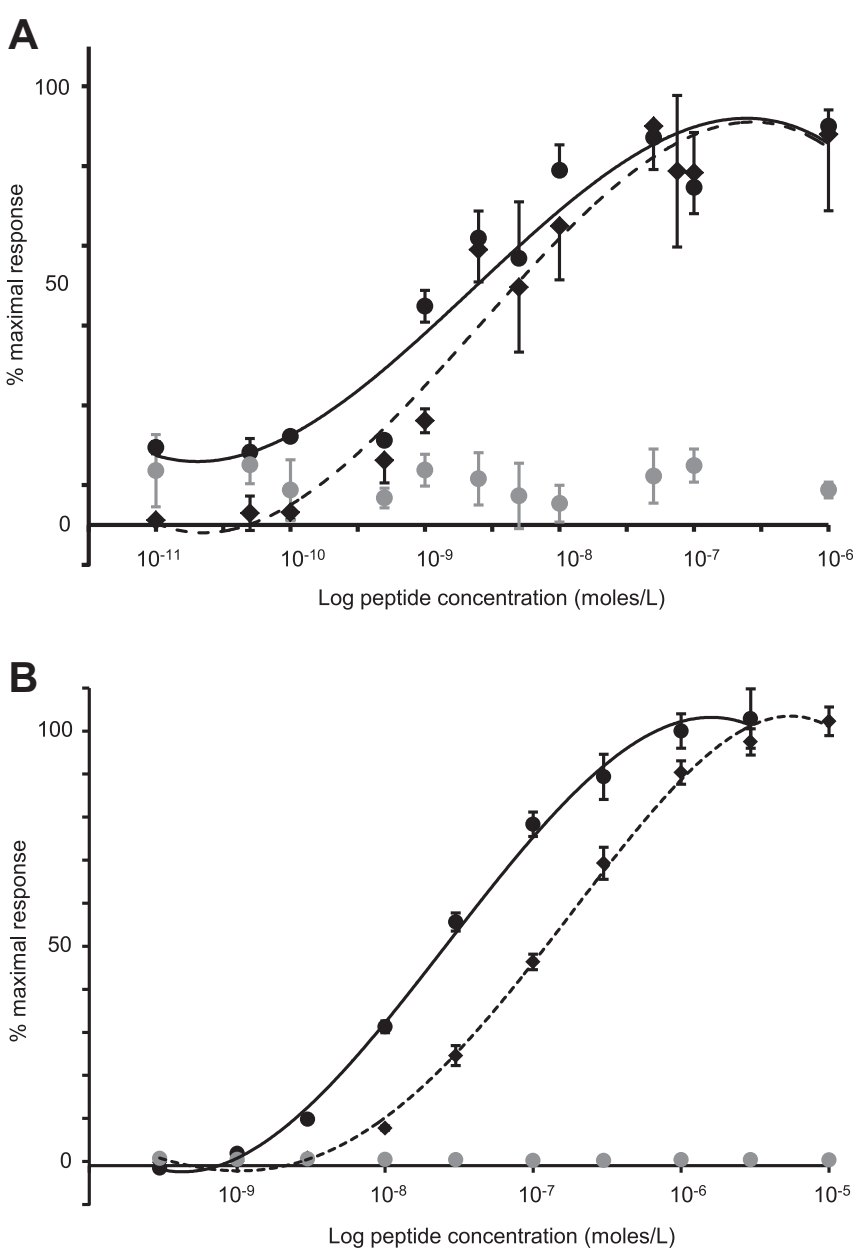

Fig. 3. The dose-response effects of peptides on the Trica-AS receptor. Grey solid circles represent the effects of Trica-AS on HEK cells containing no receptor. A) Response to Trica-AS (black solid circle) and Manse-AS (black solid diamonds) on the Trica-AS receptor (means \pm S.D, $n=3$ ), expressed in HEK cells co-transfected with $\mathrm{G} \alpha 16$, and measured using the $\mathrm{Ca}^{2+}$ reporter FLUO-4. B) The Trica-AS receptor expressed in HEK cells co-transfected with G $\alpha 15$ and Apoaequorin showing the response to Trica-AS (black solid circles), Trica-ASTCC (solid black diamonds). Means \pm S.D, $n=6$

in a dose-dependent manner, by up to $8.1 \pm 0.6$-fold in saline from tissues incubated with Trica-AS, compared with saline from control (no Trica-AS) tissues (Fig. 8).

\section{Discussion}

The PISCF/AST receptor was characterised from the red flour beetle, $T$. castaneum and shown to be activated by two PISCF/ASTs homologues (Trica-AS and Manse-AS) as well as Trica-ASTCC. This receptor shows high sequence homology to other insect PISCF/AST receptors, that have been functionally characterised from D. melanogaster (Kreienkamp et al., 2002) and mosquitoes (Mayoral et al., 2010), as well as putative PISCF/AST receptors predicted or annotated in the genomes of Apis mellifera, Nasonia vitripennis, Bombyx mori and Drosophila spp. (Mayoral et al., 2010) and A. pisum. These receptors are related to mammalian somatostatin/opioid receptors. The Trica-AS receptor (1247 bp) is shorter than the D. melanogaster (DROSTAR 1: 1401 and DROSTAR 2:1467 bp) and A. aegyptii (AeAS-CrA:1542 and AeAS-CrB: 1377 bp) orthologues, but is similar in size to human SSTR3 (1254 bp). All other human SSTRs are smaller. All these receptors share significant sequence identity in various transmembrane regions (TM1, TM2, TM3 and

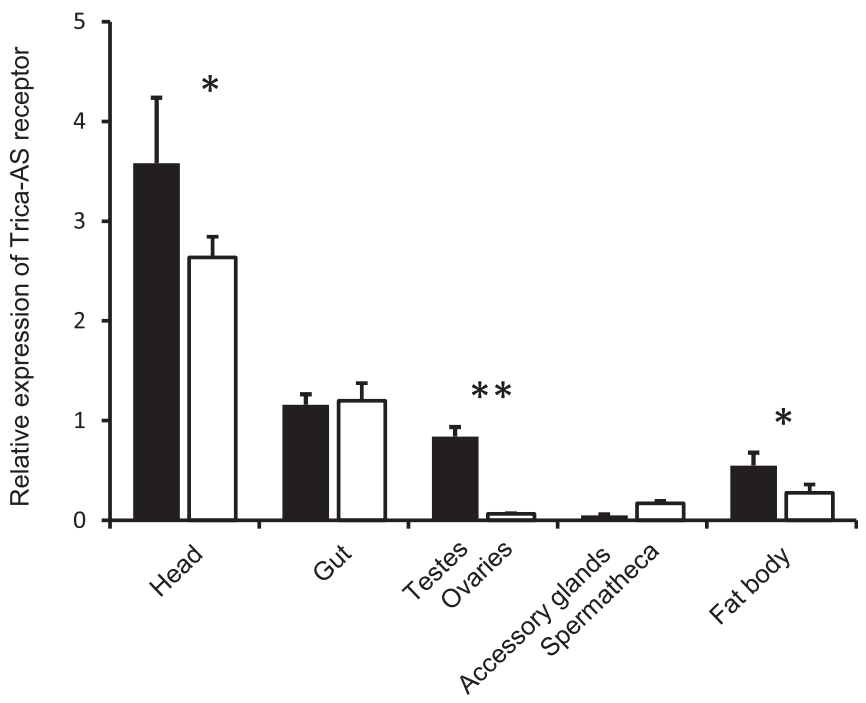

Fig. 4. Tissue distribution of the Trica-AS receptor transcript measured by qRT-PCR in adult $T$. castaneum, comparing receptor transcript levels in separate male (solid bars) and female (open bars) tissues. Mean+1- SEM from 3 biological replicates (head: 30 animals; gut, testes, ovaries, male accessory glands, female spermatheca: 50 animals; fat body: 20 animals)

TM7) and the intracellular loops involved in G-protein coupling (Mayoral et al., 2010). In Diptera, two PISCF/AST receptor paralogues are present, whereas in other orders (Coleoptera, Lepidoptera, Hymenoptera, Hemiptera) only one receptor is found (Kreienkamp et al., 2002; Mayoral et al., 2010). The reason for this difference between Diptera and other orders, and the physiological significance of two PISCF/AST receptors in flies is currently unclear. Phylogenetic analysis shows that insect PISCF/AST receptors cluster together separately from the mammalian SSTRs. Within the cluster of the insect receptors, the two receptors from the fruit fly seem to be more closely related to each other than to the mosquito receptors. This suggests that these receptors are the result of a gene duplication event that occurred after the divergence of flies and

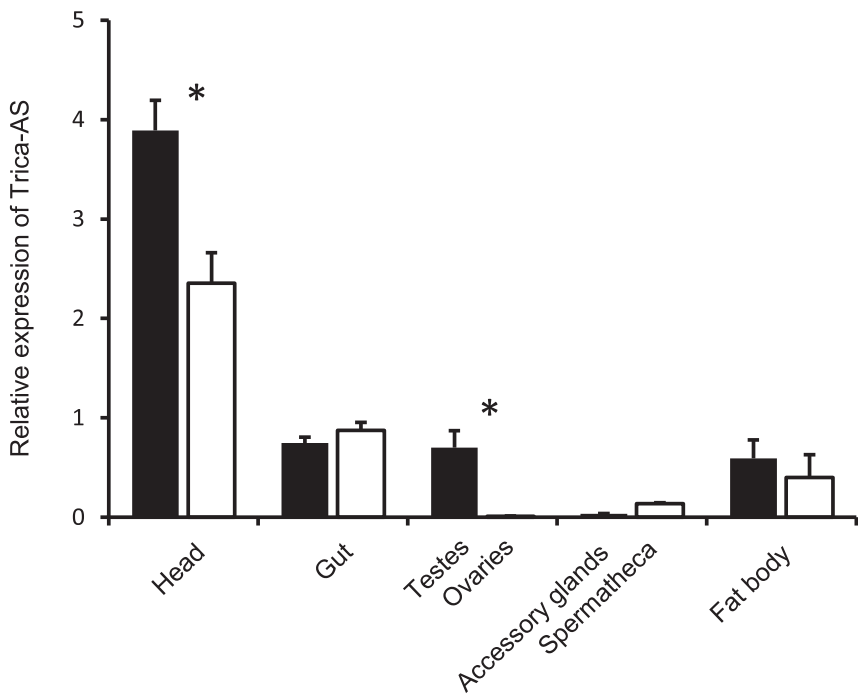

Fig. 5. Tissue distribution of the Trica-AS peptide precursor transcript measured by qRT-PCR in adult T. castaneum, comparing peptide transcript levels in separate male (solid bars) and female (open bars) tissues (Mean+/- SEM from 3 biological replicates (head: 30 animals; gut, testes, ovaries, male accessory glands, spermatheca: 50 animals; fat body: 20 animals). 

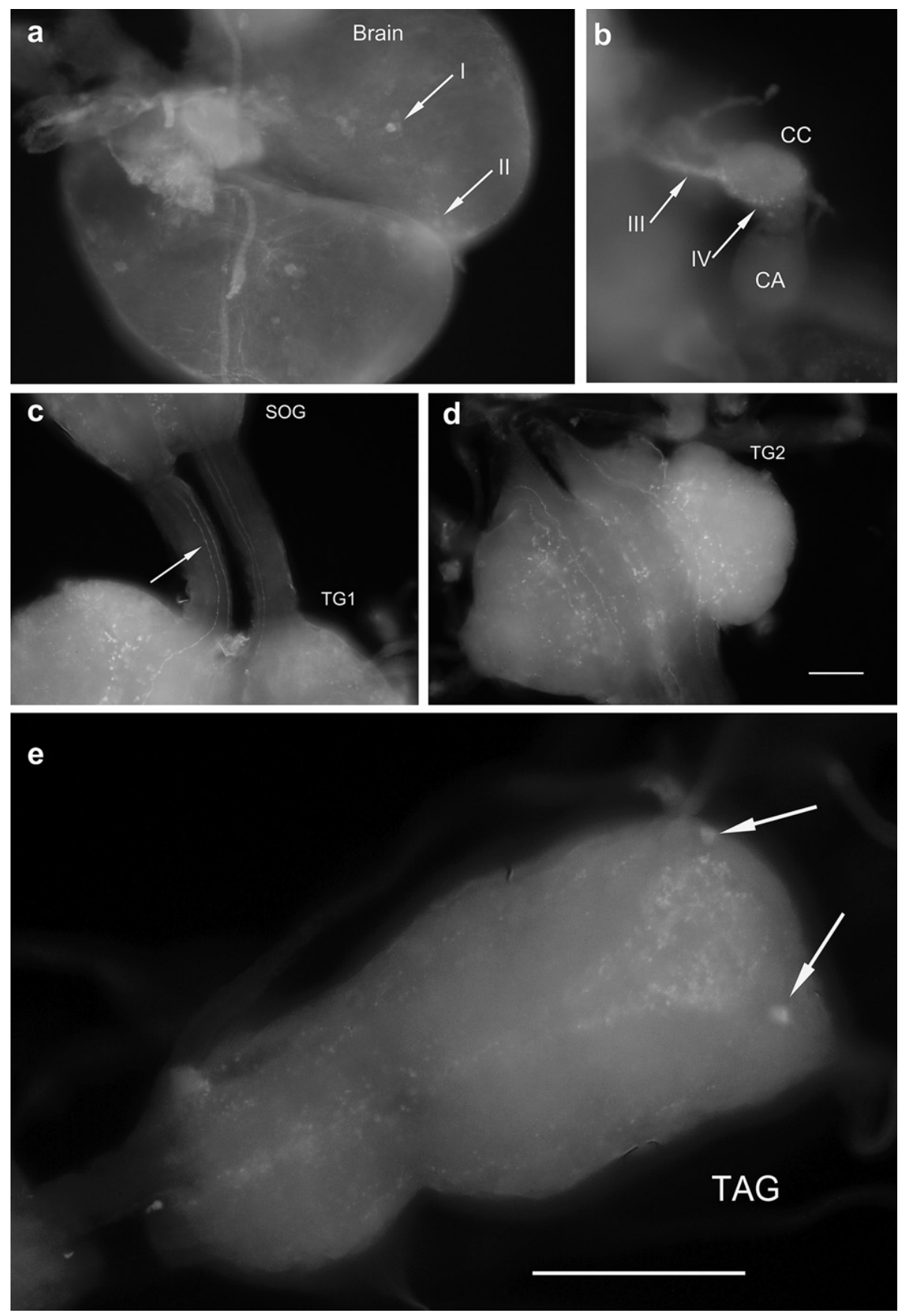

Fig. 6. Whole mount immunofluorescence in (a) the brain, (b) the retrocerebral complex and (c-e) representative ganglia from the ventral nerve cord, of adult male Tribolium castaneum. Dorsal view of the brain (a) shows the position of the two sets of perikarya (arrows I and II). Axons (arrow III) entering the corpus cardiacum (b) give rise to varicosities within the corpus cadiacum (arrow IV). Pairs of axon immunoreactive to Manse-AS antisera pass down the ventral nerve cord (c, arrow). Immunoreactive varicosities are present in all ventral ganglia $(\mathrm{c}-\mathrm{e})$, and two immunostained neurones are present in the terminal abdominal ganglion $(\mathrm{e})$. The white bar represents $100 \mu \mathrm{m}$. Abbreviations: $\mathrm{CC}=$ corpus cardiacum, $\mathrm{CA}=$ corpus allatum, $\mathrm{SOG}=$ sub oesophageal ganglion, $\mathrm{TG}=$ thoracic ganglion, $\mathrm{TAG}=$ terminal abdominal ganglion.

mosquitoes and that likely the gene which did not give rise to these receptors has been lost during fly evolution. The amino acid sequence of the Trica-AS receptor exhibits characteristic features of rhodopsin-type GPCRs, such as the seven transmembrane domains (TM1-TM7), and the conserved motif "DRY" in the second intracellular loop between the TM3 and TM4 that is often involved in the activation of the G-coupled proteins (reviewed by Vanden Broeck (1996), Vanden Broeck (2001), Mayoral et al. (2010)). Gnomon automated prediction identified two introns in the Trica-AS receptor, however, our data support the prediction reported by
Mayoral et al. (2010), that only one intron, located in the extracellular loop between TM6 and TM7 is present in this receptor.

To date, the physiological roles of Trica-AS, and hence the PISCF/ AST receptor, in beetles have received little attention. The PISCF/ ASTs have been shown to regulate juvenile hormone biosynthesis in Lepidoptera and mosquitoes, but in no other insects (reviewed by Audsley and Weaver (2009), Weaver and Audsley (2009)). In $B$. mori and A. aegypti the PISCF/AST receptors are highly expressed in the CC-CA supporting this role (Yamanaka et al., 2008; Mayoral et al., 2010). The lepidopteran PISCF/ASTs have also been shown 


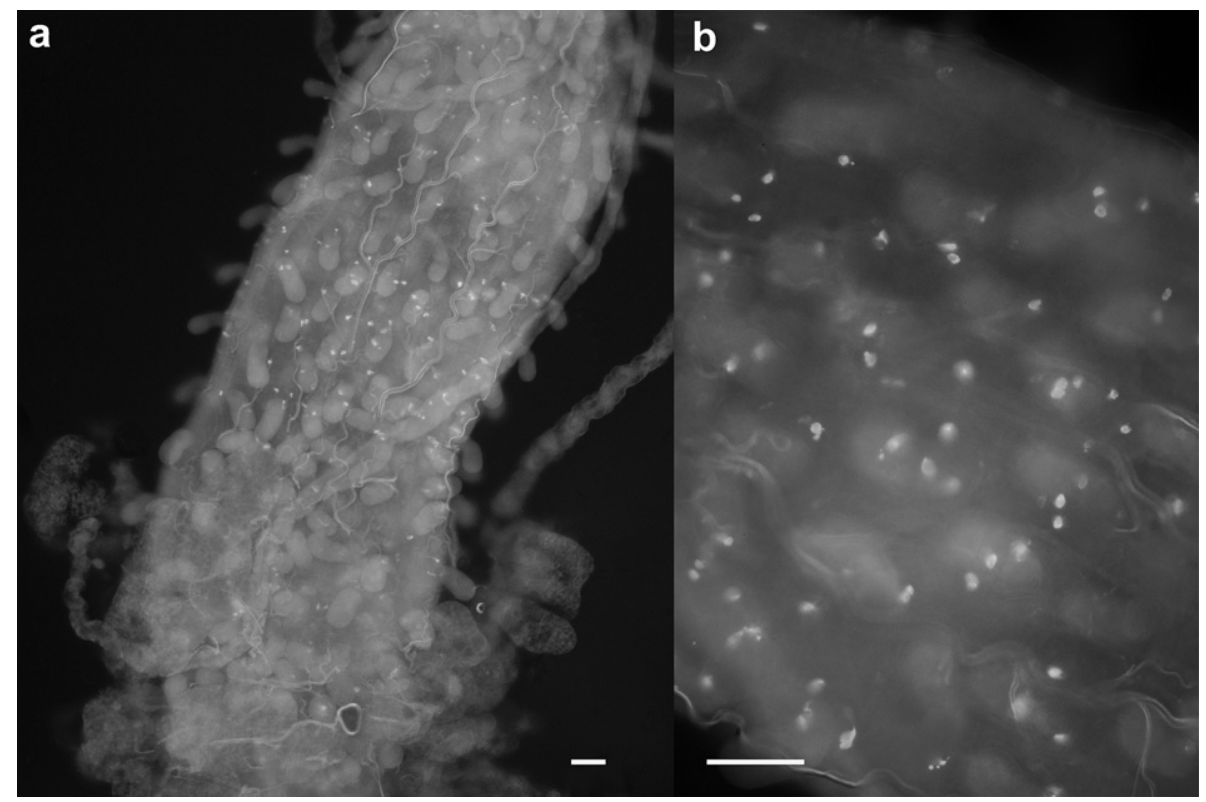

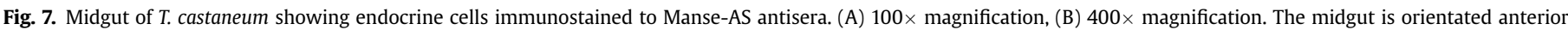
(top) to posterior (bottom). White bar $=100 \mu \mathrm{m}$.

to be immunolocalised in the CA of Heliothis virescens, Lacanobia oleracea, $M$. sexta and Spodoptera littoralis larvae, consistent with its role in regulating $\mathrm{JH}$ biosynthesis in these insects (Audsley et al., 2000; Duve et al., 2003). Homologues of PISCF/ASTs have been identified in other insects, including beetles and Drosophila spp., but they have yet to be shown to act as allatostatins in these insects (reviewed by Audsley et al. (2008), Weaver and Audsley (2009)). Yoon and Stay (1995) reported that there is no FGLa/AST-like immunoreactivity in the CA of D. melanogaster and Kreienkamp et al. (2002) reported that injection of PISCF/ASTs into second instar larvae shortened the period to pupariation. These two results led Kreienkamp et al. (2002) to suggest that a PISCF/AST, rather than a FGLa/AST is involved in JH biosynthesis in D. melanogaster.

Very little is known about the regulation of JH biosynthesis by allatoregulatory peptides in beetles, but the availability of the $T$. castaneum genome has provided the opportunity to identify the genes for these peptides and their receptors (Amare and Sweedler, 2007; Hauser et al., 2008; Li et al., 2008). Three peptide precursors, encoding an allatotropin, a PISCF/AST, and six MIP/AST peptides have been identified, whereas neither an FGLa/AST gene nor its receptor have been found (Hauser et al. 2008; Li et al., 2008;

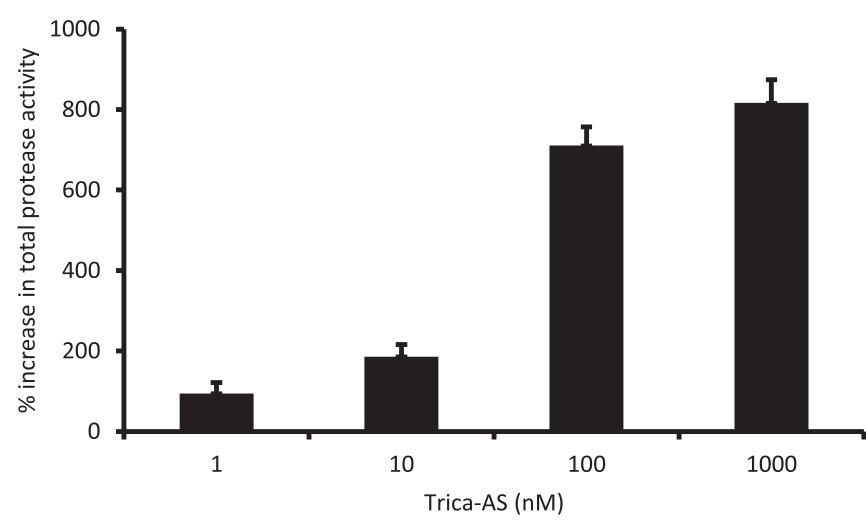

Fig. 8. The increase in protease activity of saline from the incubation of anterior midguts from Tenebrio molitor with Trica-AS, compared to control tissues. Means \pm S.E, $n=6$.
Weaver and Audsley, 2008, 2009). A peptide precursor encoding a PISCF/AST paralogue (ASTCC) was identified by Veenstra (2009), although there is no evidence to date as to whether this peptide has an allatoregulatory role. It is possible that Trica-ASTCC has similar physiological functions to Trica-AS because Trica-ASTCC is a ligand for the Trica-AS receptor. Whether Trica-ASTCC has a different functional receptor remains to be determined, but this is the first evidence that an ASTCC has a physiological role in insects. Early reports suggest that $\mathrm{JH}$ biosynthesis in beetles may not be regulated by FLGa or PISCF allatostatins (Kramer et al., 1991; Weaver and Edwards, 1998), although Weaver and Edwards (1998) did show that a number of fractions from the chromatographic separation of a T. molitor head extract did inhibit JH synthesis by the CA. More recently, Elliott et al. (2010) have provided some evidence, through bioassays and immunochemical techniques, that $\mathrm{JH}$ biosynthesis in other beetles (T. molitor and Popillia japonica) may be regulated by FGLa/ASTs. Abdel-latief and Hoffmann (2010) demonstrated that all three types of allatostatin could inhibit $\mathrm{JH}$ biosynthesis in adult female T. molitor, but the activity of individual peptides was dependent on the age of the insects and their intrinsic rate of $\mathrm{JH}$ synthesis by the CA. In day 3 adult females (high JH synthesis), Trica-AS inhibited JH III release from the CA, but activated $\mathrm{JH}$ release (low JH synthesis) from day 7 females, as did allatotropin (Trica-AT). These results demonstrate that the regulation of $\mathrm{JH}$ biosynthesis in beetles may be a complex process. Although immunoreactivity to Trica-AS was detected in the CC of T. castaneum adults, there was no evidence of immunostaining in the CA, implying that JH biosynthesis, in adult T. castaneum at least, may not necessarily be regulated by this type of allatostatin. However, the measurement of $\mathrm{JH}$ biosynthesis in the CA of T. castaneum was not undertaken in this study due, in part, to the small size of the CA/CC complex, requiring multiple glands for each assay replicate. By comparison, adult $T$. molitor are much larger overall $(6.5 \times$ longer) than $T$. castaneum (Weaver and Audsley, 2008), making them easier to dissect and enabling the determination of biosynthesis rates with single gland incubations (Abdellatief and Hoffmann, 2010).

The PISCF/ASTs are pleiotropic peptides, which is supported by the widespread distribution of these peptides and their receptors. 
However, the localisation of peptides in a particular tissue is not necessarily supported by a physiological role in that tissue, and often a role has not been investigated. In Lepidoptera, immunostaining to PISCF/AST is present in the stomatogastric nervous system, and in the nerves that supply the muscles of the foregut and midgut. Using semi-isolated preparations, Manse-AS was shown to be myoinhibitory on the foregut of lepidopteran larvae (reviewed by Audsley et al. (2008)) and Drome-AS decreases the frequency of spontaneous contractions of the foregut (crop) of adult D. melanogaster, (reviewed by Nichols et al. (2002)). In situ hybridisation also demonstrated the presence of Drome-AS mRNA in the midgut of adult flies (Price et al., 2002).

There was no evidence for PISCF/ASTs immunostaining in the stomatogastric nervous system of adult $T$. castaneum, and Trica-AS had no effect on the spontaneous contractions of the hindgut of adults or larvae. Unlike lepidopteran larvae, the foregut of $T$. castaneum does not appear to contract spontaneously, and hence it was not possible to determine any potential inhibition of contractions of this region of the gut by Trica-AS. However, the immunolocalisation of Trica-AS in the midgut endocrine cells, the expression of both the Trica-AS receptor and peptide in the gut, along with the characterisation of the Trica-AS receptor from larval gut tissues, all suggest that Trica-AS has a role on the gut of T. castaneum. This role would appear to be the stimulation of the release of proteases from the midgut, because total protease activity in the anterior midgut of T. molitor was significantly greater in saline from tissues incubated with Trica-AS than in saline from control (no peptide) tissues. The specific proteases released were not investigated. Similarly, insect myosuppressins and sulfakinins stimulate protease release from the gut of the scallop, Pecten maximus, and the red palm weevil, Rhynchophorus ferrugineus (Nachman et al., 1997), whereas leucokinins inhibit protease release from the gut of larvae of the moth Opisina arenosella (Harshini et al., 2002). Transcripts for the Trica-AS receptor and peptide were also found in reproductive organs and fat body.

There is widespread distribution of Trica-AS in the central nervous system, and immunoreactive neurones are present in both the lateral and median neurosecretory cells of the brain. Nerves from these cells (NCCI and NCCII) pass through the brain to the retrocerebral complex. This is clearly demonstrated in larval Lepidoptera, where PISCF/AST (Manse-AS) has been immunostained in the neurosecretory cells of the brain, and the NCCI and NCCII, as well as in the CC and CA (reviewed by Audsley et al. (2008)). A similar distribution of PISCF/AST has been shown in D. melanogaster and A. aegypti (Merte and Nichols, 2002; Kreienkamp et al., 2002; Mayoral et al., 2010). Although immunostaining to PISCF/AST was detected in neurosecretory cells of the brain and in the CC of T. castaneum, there was no detectable PISCF/AST-like immunoreactivity in the CA. In the beetles P. japonica and T. molitor, FGLa/AST shows a similar distribution, present in brain neurosecretory cells and in the $\mathrm{CC}$, but not the CA, although immunostained nerves running over the surface of the CA of $P$. japonica were observed (Elliott et al., 2010).

Although immunostaining to PISCF/AST was widespread in the ventral nerve cord and in the suboesophageal ganglion (SOG), the only neurones that appeared to be immunostained were in the terminal abdominal ganglion. No other immunostained neurones were visualised with the methods used in this study, but each ganglion did contain numerous immunostained varicosities. Similar varicosities were reported for immunostaining to a vasopressin-like peptide (AVPL) in the ventral nerve cord of T. castaneum, where only two anti-AVPL positive cells in the central nervous system (in the SOG), were identified (Aikins et al., 2008).

In summary, the somatostatin-like peptide receptor has been structurally characterised from the beetle, T. castaneum, and shown to be activated by insect PISCF/ASTs and Trica-ASTCC, but not other allatoregulatory peptides or somatostatin ${ }^{14}$, using a heterologous assay. The receptor, and/or its ligand (Trica-AS) are expressed in a wide variety of tissues, including the gut, brain and reproductive organs. In contrast to ASTs in some insects, Trica-AS does not appear to be myo-inhibitory on the gut in T. castaneum, but does stimulate protease release from the anterior midgut, implying a role in the digestive process. Although Trica-AS has previously been shown to inhibit JH synthesis by the CA in another tenebrionid beetle, T. molitor (Abdel-latief and Hoffmann, 2010) the exact physiological role of the receptor and peptide within the CA of T. castaneum remains to be determined.

\section{Acknowledgements}

Funding for this work was provided by the Chemicals Regulation Directorate, Health and Safety Executive, UK, on behalf of Defra, the Interuniversity Attraction Poles program [Belgian Science Policy Grant (P6/14)], the Flemish (FWO) and the KU Leuven (GOA/11/02) Research Foundations for financial support. HPV and KV were both supported by a FLOF fellowship of the KU Leuven. Y-JK was supported by the National Research Foundation of Korea (Grant 20090089247) and the Gwangju Institute of Science and Technology (Basic Research Projects in High-tech Industrial Technology 2012).

The authors thank Joost Van Duppen and Nick Suetens for technical assistance.

\section{Appendix A. Supplementary data}

Supplementary data related to this article can be found at http:// dx.doi.org/10.1016/j.ibmb.2012.09.007.

\section{References}

Abdel-latief, M., Hoffmann, K.H., 2010. Neuropeptide regulators of the juvenile hormone biosynthesis (in vitro) in the beetle Tenebrio molitor (Coleoptera, Tenebrionidae)Arch. Insect Biochem. Physiol. 74, 135-146.

Aikins, M.J., Schooley, D.A., Begum, K., Detheux, M., Beeman, R.W., Park, Y., 2008. Vasopressin-like peptide and its receptor function in an indirect diuretic signaling pathway in the red flour beetleInsect Biochem. Mol. Biol. 38, 740-748.

Amare, A., Sweedler, J.V., 2007. Neuropeptide precursors in Tribolium castaneumPeptides 28, 1282-1291.

Audsley, N., Weaver, R.J., 2009. Neuropeptides associated with the regulation of feeding in insectsGen. Comp. Endo 162, 93-104.

Audsley, N., Duve, H., Thorpe, A., Weaver, R.J., 2000. Morphological and physiological comparisons of two types of allatostatin in the brain and retrocerebral complex of the tomato moth, Lacanobia oleracea, (Lepidoptera: Noctuidae) J. Comp. Neurol. 424, 37-46.

Audsley, N., Matthews, H.J., Price, N.R., Weaver, R.J., 2008. Allatoregulatory peptides in Lepidoptera, structures, distribution and functionsJ. Insect Physiol. 54, 969-980.

Coast, G.M., Schooley, D.A., 2011. Toward a consensus nomenclature for insect neuropeptide and peptide hormonesPeptides 32, 620-631.

Down, R.E., Matthews, H.J., Audsley, N., 2010. Effects of Manduca sexta allatostatin and an analogue on the pea aphid Acyrthosiphon pisum (Hemiptera: Aphididae) and degradation by enzymes from the aphid gutPeptides 31, 489-497.

Duve, H., Audsley, N., Weaver, R.J., Thorpe, A., 2003. Allatostatins and allatotropin in the corpus cardiacum/corpus allatum complex of larval and adult lepidopterans studied by confocal laser scanning microscopy: correlation to juvenile hormone biosynthesisCell Tissue Res. 314, 281-295.

Elliott, K.L., Chan, K.K., Stay, B., 2010. Evidence for a Phe-Gly-Leu-amide-like allatostatin in the beetle Tenebrio molitorPeptides 31, 402-407.

Harshini, S., Nachman, R.J., Sreekumar, S., 2002. Inhibition of digestive enzyme release by neuropeptides in larvae of Opisina arenosella (Lepidoptera: Cryptophasidae)Comp. Biochem. Physiol. 132, 353-358.

Hauser, F., Cazzamali, G., Williamson, M., Park, Y., Li, B., Tanaka, Y., Predel, R., Neupert, S., Schachtner, J., Verleyen, P., Grimmelikhuijzen, C.J.P., 2008. A genome-wide inventory of neurohormone GPCRs in the red flour beetle Tribolium castaneumFront. Neuroendocrinol. 29, 142-165.

Kramer, S.J., Toschi, A., Miller, C.A., Kataoka, H., Quistad, G.B., Li, J.P., Carney, L., Schooley, D.A., 1991. Identification of an allatostatin from the tobacco hornworm Manduca sextaProc. Nat. Acad. Sci. USA 88, 9458-9462. 
Kreienkamp, H.-J., Larusson, H.J., Witte, I., Roeder, T., Birgul, N., Honck, H.-H., Harder, S., Ellinghausen, G., Buck, F., Richter, D., 2002. Functional annotation of two orphan G-protein-coupled receptors, Drostar1 and -2, from Drosophila melanogaster and their ligands by reverse pharmacologyJ. Biol. Chem. 277 (42), 39937-39943.

Li, B., Predel, R., Neupert, S., Hauser, F., Tanaka, Y., Cazzamali, G., Williamson, M. Arakane, Y., Verleyen, P., Schoofs, L., Schachtner, J., Grimmelikhuijzen, C.J.P., Park, Y., 2008. Genomics, transcriptomics, and peptidomics of neuropeptides and protein hormones in the red flour beetle Tribolium castaneumGenome Res. $18,113-122$.

Matthews, H.J., Audsley, N., Weaver, R.J., 2008. In vitro and in vivo effects of myoactive peptides on larvae of the tomato moth Lacanobia oleracea and the cotton leaf worm Spodoptera littoralis (Lepidoptera; Noctuidae)Arch. Insect Biochem. Physiol. 69, 60-69.

Matthews, H.J., Down, R.E., Audsley, N., 2010. Effects of Manduca sexta allatostatin and an analogue on the peach-potato aphid Myzus persicae (Hemiptera: Aphididae) and degradation by enzymes in the aphid gutArch. Ins Biochem. Physiol. 75, 139-157.

Mayoral, J.G., Nouzova, M., Brockhoff, A., Goodwin, M., Hernandez-Martinez, S. Richter, D., Meyerhof, W., Noriega, F.G., 2010. Allatostatin-C receptors in mosquitoesPeptides 31, 442-450.

Merte, J., Nichols, R., 2002. Drosophila melanogaster myotropins have unique functions and signaling pathwaysPeptides 23, 757-763.

Moller, L.N., Stidsen, C.E., Hartmann, B., Holst, J.J., 2003. Somatostatin receptorsBiochim. Biophys. Acta Biomembranes 1616, 1-84.

Nachman, R.J., Giard, W., Favrel, P., Suresh, T., Sreekumar, S., Holman, G.M., 1997. Insect myosuppressins and sulfakinins stimulate release of the digestive enzyme $\alpha$-Amylase in two invertebrates: the scallop Pecten maximus and insect Rhynchophorus ferrugineusAnn. N.Y. Acad. Sci. 814, 335-338.

Nichols, R., Bendena, W.G., Tobe, S.S., 2002. Myotropic peptides in Drosophila melanogaster and the genes that encode themJ. Neurogenetics 16, 1-28.
Offermanns, S., Simon, M.I., 1995. G alpha 15 and G alpha 16 couple a wide variety of receptors to phospholipase CJ. Biol. Chem. 270 (25), 15175-15180.

Price, M.D., Merte, J., Nichols, R., Koladich, P.M., Tobe, S.S., Bendena, W.G., 2002 Drosophila melanogaster flatline encodes a myotropin orthologue to Manduca sexta allatostatinPeptides 23, 784-794.

Vanden Broeck, J., 1996. G protein-coupled receptors in insect cellsInt. Rev. Cytol. $164,189-268$.

Vanden Broeck, J., 2001. Insect G protein coupled receptors and signal trans ductionArch. Insect Biochem. Physiol. 48, 1-12.

Vandesompele, J., De Preter, K., Pattyn, F., Poppe, B., Van Roy, N., De Paepe, A., Speleman, F., 2002. Accurate normalization of real-time quantitative RT-PCR data by geometric averaging of multiple internal control genesGenome Biol. 3 (7):research0034.1

Veenstra, J.A., 2009. Allatostatin C and its paralog allatostatin double C: the arthropod somatostatinsInsect Biochem. Mol. Biol. 39, 161-170.

Weaver, R.J., Audsley, N., 2008. Neuropeptides of the beetle, Tenebrio molitor identified using MALDI-TOF mass spectrometry and deduced sequences from the Tribolium castaneum genomePeptides 29, 168-178.

Weaver, R.J., Audsley, N., 2009. Neuropeptide regulators of juvenile hormone synthesis. Structures, functions, distribution, and unanswered questions. Trends in Comparative Endocrinology and NeurobiologyAnn. N.Y. Acad. Sci. 1163, 316-329.

Weaver, R.J., Edwards, J.P., 1998. Neuropeptide inhibitors of juvenile hormone synthesis in the mealworm and tomato moth. Trends in Comparative Endocrinology and NeurobiologyAnn. N.Y. Acad. Sci. 839, 450-452.

Yamanaka, N., Yamamoto, S., Zitnan, D., Watanabe, K., Kawada, T., Satake, H. Kaneko, Y., Hiruma, K., Tanaka, Y., Shinoda, T., Kataoka, H., 2008. Neuropeptide receptor transcriptome reveals unidentified neuroendocrine pathwaysPLOS ONE 3, e3048.

Yoon, J.G., Stay, B., 1995. Immunocytochemical localization of Diploptera punctata allatostatin-like peptide in Drosophila melanogasterJ. Comp. Neurol. 363, 475-488. 